\title{
Design and evaluation of rhubarb total free anthraquinones oral colon-specific drug delivery granules to improve the purgative effect
}

\author{
Hongwei Wei ${ }^{1}$, Jinhua Chang, Pei Liu ${ }^{1}$, Zhongsi Li ${ }^{1}$, Guangxin Miao ${ }^{1}$, Xigang Liu ${ }^{1}$, Cuizhe Liu ${ }^{1 *}$, \\ Xiangrong Zhang ${ }^{2}$
}

${ }^{1}$ Hebei Province key Laboratory of Research and Development for Chinese Medicine, Chengde Medical College, Chengde, Hebei, P. R. China, ${ }^{2}$ Shenyang Pharmaceutical University, Shenyang, Liaoning, P. R. China

\begin{abstract}
Rhubarb is commonly used as a cathartic in Asian countries. However, researchers have devotedextensive concerns to the quality control and safety of rhubarb and traditional Chinese preparations composed of rhubarb due to the instable purgative effect and potential nephrotoxicity of anthraquinones. In this study, we aimed to prepare rhubarb total free anthraquinones (RTFA) oral colon-specific drug delivery granules (RTFA-OCDD-GN) to delivery anthraquinones to colon to produce purgative effect. RTFAOCDD-GN were prepared using chitosan and Eudragit S100 through a double-layer coating process and the formulation was optimized. Continuous release studies were performed in a simulated gastric fluid

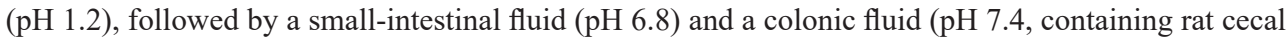
contents). The purgative effect test was performed in rats. The dissolution profile of RTFA-OCDD-GN showed that the accumulative dissolution rate of RTFA was about $83.0 \%$ in the simulated colonic fluid containing rat cecal contents and only about $9.0 \%$ in the simulated gastrointestinal fluids. And the RTFAOCDD-GN could produce the comparative purgative activity as rhubarb, suggesting it could deliver the free AQs to the colon. The RTFA-OCDD-GN was a useful media to enhance the purgative activity of free anthraquinones after administered orally.
\end{abstract}

Keywords: Rhubarb. Anthraquinones. Oral colon-specific drug delivery granules. Dissolution. Purgative activity.

\section{INTRODUCTION}

Rhubarb, a well-known Chinese herbal medicine, has been widely used for thousands of years in China due to its purgative activity (Fu et al., 2011). As one of main active constituents of rhubarb, anthraquinones (AQs) are usually considered as the chemical basis of purgative activity of rhubarb. AQs exist both in free and conjugated forms, and the most is conjugated AQs in rhubarb medical material (RMM). Free AQs consisted of aloe-emodin, rhein, emodin, chrysophanol and physcione. Conjugated AQs are glucoside of free AQs with a $\beta$-glucosidic bond (Fu et al., 2011; Wang, 1991; Yi et al., 2011) (the chemical structure of AQs was shown in Scheme 1). The conjugated

\footnotetext{
*Correspondence: C. Liu. Hebei Province Key Laboratory of Research and Development for Chinese Medicine, Chengde Medical College, Chengde, Hebei 067000, P. R. China. Tel: +86 0314-2290359 / Fax: +86 0314-2290359; E-mail: liucuizhexy@163.com

The first two authors contributed equally to this work.
}

AQs, which bear $\beta$-glucosidic bonds, can avoid being hydrolyzed by $\alpha$-glucosidase in the upper GI tract, then reach colon and be hydrolyzed to free AQs by bacteriaproducing $\beta$ - glucosidase stimulating purgation $(\mathrm{Wu}$, 2002; Min et al., 2008; Yang, Wang, Zhang, 2012). Hence the free AQs are the ultimate substance playing such a purgative action as demonstrated in the previous studies (Wu, 2002; Yamauchi et al., 1992). The mechanism of purgative activity produced by free AQs is to stimulate large intestinal paries and nervous plexus to promote intestinal peristalsis and reduce water absorption at colon (Rauwald, 1998; Yang et al., 2011). However, free AQs can be rarely delivered to colon due to the absorption or destruction in upper gastrointestinal (GI) tract after oral administration. Hence the rhubarb containing conjugated AQs is used to exert purgative effect in clinic.

Chinese Pharmacopoeia records a total of 104 types of rhubarb-containing traditional Chinese medicines (TCM). In these TCM, rhubarb exerts the main purgative 


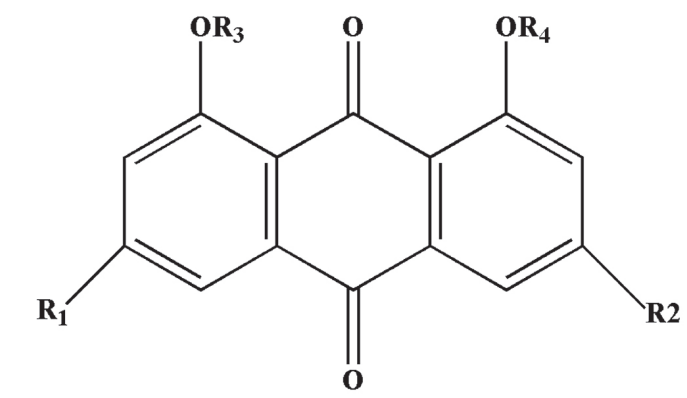

Combined AQs: $\mathbf{R}_{\mathbf{3}}=\mathbf{H}, \mathbf{R}_{\mathbf{4}}=\mathrm{Glc} ; \mathbf{R}_{\mathbf{3}}=\mathrm{Glc} ; \mathbf{R}_{\mathbf{4}}=\mathrm{H}$

Free AQs: $\quad R_{1} \quad R_{2}$

Chrysophanol: $\mathrm{H} \quad \mathrm{CH}_{3}$

Aloe-emodin: $\mathrm{H} \quad \mathrm{CH}_{2} \mathrm{OH}$

Rhein: $\quad \mathrm{H} \quad \mathrm{COOH}$

Emodin: $\quad \mathrm{OH} \quad \mathrm{CH}_{3}$

Physcion $\quad \mathrm{OCH}_{3} \quad \mathrm{CH}_{3}$

SCHEME 1 - Chemical structures of AQs and AQs glycosides.

activity. However, conjugated AQs are easily hydrolyzed to free AQs in the process of extracting rhubarb, which resulted in the attenuation of purgative activity of rhubarb free AQs extract (Wu, 2002; Sun et al., 2006). Therefore, the rhubarb used in the TCM is in the form of either all or partial crude powder. Due to the difference on field, cultivation and harvesting time, rhubarb and these rhubarb-containing TCM exhibited instable purgative effect. Such as San-Huang tablet (SHT), it contains rhubarb and has been used popularly to treat constipation in clinic. However, it exhibited some defects, such as large oral dosage, instable purgative effect and so on. To overcome these problems, San-Huang dispersible tablet (SHDT) is successfully prepared in our previous study. In the formulation of SHDT, rhubarb total free AQs (RTFA) extract is used instead of rhubarb crude power and rough extract, and then is granulated by the enteric polymer. The pharmacodynamic study results indicated that SHDT exerted strong and stable purgative effect compared with SHT (Liu et al., 2009).

In recent years, more and more studies have reported that emodin and other AQs can induce renal toxicity after oral administration (Keiyu et al., 2011; National Toxicology Program, 2001). These studies have promoted researchers to think about the safety of rhubarb and the preparations containing rhubarb. Interestingly, the nephrotoxicity caused by processed rhubarb was attenuated in comparison with rhubarb due to the reduction of conjugated AQs in the processed rhubarb (Wang et al., 2009). Moreover, the in situ enteric absorption kinetics of free AQs demonstrated that most of free AQs were absorbed in the small intestinal and only a little part were absorbed in colon (Liu et al., 2011). Therefore, we presented a hypothesis that RTFA extract is used as the alternative of rhubarb and the almost all free AQs contained in the RTFA extract is delivered to colon that can contribute to improve the purgative effect and reduce the nephrotoxicity.

Colon-specific drug delivery system (OCDDS) can prevent drug from being absorbed in the upper GI region and then promptly release into the proximal colon (Italia et al., 2007; Klan, Prebeg, Kurjakovic, 1999; Piao, Lee, Lee, 2008). Various approaches have been used for oral delivery of drug to the colon, which include time-dependent system, $\mathrm{pH}$-dependent system and bacteria-dependent delivery. However, the OCDDS with single transmit system has some defects, such as being easily effected by food and GI tract $\mathrm{pH}$ and movement, further leading to the release profiles with a poor reproducibility (Ashford et al., 1993; Moore, Flanner, 1996). Thus the combination of two principles is usually proposed to obtain satisfactory OCDDS. Many studies have demonstrated that chitosan can be used as the carrier to prepare OCDDS due to its low toxicity, good biocompatibility and biodegradability (Tozaki et al., 1997; Tozaki et al., 2002). However, chitosan can be dissolved in acidic solution. Therefore, an outer enteric coating is essential to prevent the early breakdown of OCDDS prepared with chitosan in the stomach (Khan, Prebeg, Kurjakovic, 1999). Eudragit S100 and Eudragit L100 are dissolved in aqueous medium above $\mathrm{pH}$ 6.8, hence the polymers are commonly used as the carriers to prepare the $\mathrm{pH}$-dependent OCDDS (Kaur, Kim, 2009). So this paper aimed to prepare RTFA oral colonspecific drug delivery granules (RTFA-OCDD-GN) using chitosan and $\mathrm{pH}$-dependent Eudragit polymers as carriers by a double-layer coating process to deliver the free AQs contained in the RTFA extract to colon releasing. Moreover, in vitro dissolution of this newly developed drug delivery system were studied, and the in vivo purgative activity was compared with RMM.

\section{MATERIAL AND METHODS}

\section{Materials}

Dried root and rhizoma of Rheum officinale Baill. of the Polygonaceae family were purchased from Beijing TongRenTang Drugstore and identified by Professor Chunying Zhao, a botanist at Chengde Medical College (Hebei, China). Methacrylic acid copolymers (Eudragit S100) and Eudragit L100 were purchased from Changwei Medicine Corporation (Shanghai, China), and 
polyethylene glycol-6000 (PEG-6000) was purchased from Zhengxing Chemical Industry Research Institute (Suzhou, china). Chitosan (viscosity of $100 \mathrm{cps}$ ) was purchased from Fuchen Chemical Industry Company (Tianjin, China). Hydroxypropyl methyl cellulose (HPMC) and microcrystalline cellulose (MCC) were purchased from Zhanwang Medicine Limited Company (Huzhou, China). Glycerinum and diethyl phthalate (DEP) and castor oil and lauryl sodium sulfate (SDS) was obtained from Jiaxing Chemical Industry Co., Ltd. (Tianjin, China). Methyl alcohol of chromatogram grade was purchased from Xiehehaopeng Chromatogram Science \& Technology Co., Ltd. (Tianjin, China). Other chemicals and solvents were of analytical reagent grade, and double-distilled water was used throughout the study.

\section{Animals}

Sprague Dawley (SD) rats weighing 180-240 g were provided by Tianjin Shanchuanhong Laboratory Animal Science \& Technology Co., Ltd. (License No. SCXK 2009-0001). All animal experiments were performed in accordance with the Guide for the Care and Use of Laboratory Animals, as adopted and promulgated by the Ministry of Science and Technology of China.

\section{Extraction of rhubarb}

The rhubarb was extracted according to the method (Li et al. (2009)). Briefly, rhubarb was extracted by $30 \%$ ethyl alcohol three times and then the filtrate was concentrated under vacuum. The concentrated liquid was added in the column of anion exchange resin and eluted. Then the eluant was collected, concentrated and filtered. The filtrate was dried in oven at $40^{\circ} \mathrm{C}$. The dried residue was the RTFA extract, in which the AQs existed only in free form and the content of RTFA determined by HPLC (Liu et al., 2005) was 54.5\%.

\section{Core drug-loaded granules preparation}

RTFA extract (smaller than $150 \mu \mathrm{m})$ was mixed with PEG-6000 and MCC (smaller than $150 \mu \mathrm{m})(1: 0.6: 4$, $w: w: w)$. Then the mixture was granulated using $9 \mathrm{~mL}$ of aqueous solution containing 2\% (w:v) HPMC and 2\% $(w: v)$ PEG-6000 as adhesive with high-speed stirring machine (Song Qing Machinery Factory, China). The granules were oven-dried at $50^{\circ} \mathrm{C}$ and screened through a 50-mesh sieve to obtain core drug-loaded granules. The formulation was screened with the accumulative dissolution rate of RTFA in $\mathrm{pH} 7.4$ medium as an index and the optimized formulation of core drug-loaded granule was listed in Table I.

TABLE I - The formulation of core drug-loaded granules

\begin{tabular}{lc}
\hline Ingredient & Amount (g, mL) \\
\hline RTFA extract (the content of & 17.9 \\
RTFA is 54.5\%) & 71.6 \\
MCC & 10.5 \\
PEG-6000 & 32 \\
2\% (w/v) aqueous solvent of & 100 \\
HPMC and PEG-6000 & \\
Core drug-loaded granules & \\
\hline
\end{tabular}

\section{Granule coating}

\section{Chitosan subcoating}

Firstly, chitosan was dissolved in $1 \% \mathrm{HCl}$ aqueous solution (Orienti et al., 2002) at a concentration of $1.20 \%$ $(w: v)$ and stirred overnight prior to coating. Then, glycerin, as the plasticizer of chitosan subcoating formulation, equal to $20 \%$ weight of the dry polymer, was added in the chitosan solution stirring for $1 \mathrm{~h}$ to prepare the subcoating fluid. The subcoating formulation was listed in Table II. One hundred gram of core drug-loaded granules were coated with $420 \mathrm{~mL}$ chitosan sub-coating fluid by a fluid bed coating apparatus equipped with a bottom sprayer. Briefly, the coating process was performed using following parameters: inlet temperature of $80^{\circ} \mathrm{C}$, product temperature of $55^{\circ} \mathrm{C}$, spray rate of $1.0-1.6 \mathrm{rpm}$, atomizing air pressure of $0.08-0.1 \mathrm{MPa}$ and nozzle diameter of 0.5 $\mathrm{mm}$. The subcoating granules were further fluidized for $20 \mathrm{~min}$ and oven-dried at $40^{\circ} \mathrm{C}$ for $2 \mathrm{~h}$. The chitosan subcoating formulation was screened with the dissolution profile of sub-coating granule in simulated small intestinal fluid for $4 \mathrm{~h}$.

TABLE II - Chitosan subcoating formulation

\begin{tabular}{lc}
\hline Ingredient & Amount (g, mL) \\
\hline chitosan & 1.25 \\
glycerin & 0.312 \\
$\begin{array}{l}\% \text { hydrochloric aqueous } \\
\text { solution }\end{array}$ & 100 \\
\hline
\end{tabular}

\section{Eudragit S100 coating}

Firstly Eudragit S100 was dissolved in 95\% ethanol aqueous solution, and then the polymer solution 
was mixed with DEP (equal to $20 \%$ weight of the dry polymer) and $3 \%(w / v)$ of talcum, and finally the mixture was stirred for $24 \mathrm{~h}$ to obtain the uniform Eudragit S100 coating fluid. The Eudragit S100 coating formulation was listed in Table III. In the formulation, DEP was used as the plasticizer and talcum was used as antisticking agent. Subsequently, the $100 \mathrm{~g}$ of sub-coating granules were coated with $350 \mathrm{~mL}$ Eudragit S100 coating fluid. The coating process was performed using following parameters: inlet temperature of $50^{\circ} \mathrm{C}$, product temperature of $40^{\circ} \mathrm{C}$, spray rate of $1.2-2.0 \mathrm{rpm}$, atomizing air pressure of 0.05-0.07 MPa and nozzle diameter of 0.5 $\mathrm{mm}$. Double-layer coated granules were further fluidized for $20 \mathrm{~min}$, oven-dried at $40^{\circ} \mathrm{C}$ for $2 \mathrm{~h}$ and then sieved at 40-60 mesh. The double layer coating granules obtained was RTFA-OCDD-GN. The content of RTFA determined by HPLC (Liu et al., 2005) was $8.49 \%$ in RTFA-OCDDGN. The Eudragit S100 coating formula was screened by the accumulative dissolution rate of RTFA in simulated stomach and small intestinal fluid.

TABLE III - Enteric coating formulation

\begin{tabular}{lc}
\hline Ingredient & Amount $(\mathbf{g}, \mathbf{m L})$ \\
\hline Eudragit $\AA \mathrm{S} 100$ & 5 \\
DEP & 1 \\
talc & 3 \\
$95 \%$ ethanol solution & 100 \\
\hline
\end{tabular}

\section{Factorial design}

According to the results of signal factor, the dissolution profile of RTFA-OCDD-GN was affected by the amount of chitosan formulation and Eudragit S100 formulation respectively. The factorial design was constructed by statistical software (SPSS. 19.0) to investigate the interaction of factor A (the amount of chitosan formulation) and factor B (the amount of Eudragit $\mathrm{S} 100)$. The data was analyzed using the variance index $L$ which is calculated by the weighted grading method with the equation as following:

$$
L=\left|L_{1}-0 \%\right| \times 100 \times 1+\left|L_{2}-80 \%\right| \times 100 \times 2
$$

In the equation (1), the $L_{l}$ expressed the dissolution rate in the simulated gastric and small intestinal fluid (the weight was 1), the $L_{2}$ expressed the dissolution rate in the simulated colonic fluid (the weight was 2). The factor levels were presented in Table IV. Nine experimental runs were formulated for RTFA-OCDD-GN.

\section{The reproducibility of optimized formulation}

Three batches RTFA-OCDD-GN were prepared with the optimized formulation. The reproducibility was evaluated using the method of similarity factor, with the accumulative dissolution rates of RTFA in the simulated gastric, small intestinal and colonic fluid as indexes. The similarity factor $\left(f_{2}\right)$, a method recommended by FDA, can be used to evaluate the comparability among different batches of preparations objectively. The $f_{2}$ is calculated with the equation (2) as following:

$$
f_{2}=50 \times \lg \left\{\left[1+1 / n \sum(Y R t-Y T t)^{2}\right]^{-0.5} \times 100\right\}
$$

In the equation (2), YRt and YTt expressed the accumulative dissolution rate at different time of reference and test preparation respectively; the $n$ expressed the numbers of withdrawing samples. The FDA provides that the two dissolution curves is completely coincident when $f_{2}$ is equal to 100 , similar when $f_{2}$ is between 50 to 100 and not similar when $f_{2}$ is below 50 .

The reference dissolution curve of RTFA-OCDDGN was defined in the paper as following: the accumulative dissolution rate of RTFA in the simulated gastric fluid for $2 \mathrm{~h}$ was approximate $0 \%$, and it was below $10 \%$ in the simulated small intestinal fluid for $4 \mathrm{~h}$, and it was more than $80 \%$ in the simulated colonic fluid for $4 \mathrm{~h}$.

\section{Dissolution test}

The dissolution test was performed in triplicate using dissolution apparatus (Tian Da Tian Fa Science \& Technology Co., Ltd., Tianjin, China) at $37^{\circ} \mathrm{C}$ with a stirring speed of $75 \mathrm{rpm}$ according to the Chinese Pharmacopoeia method with some minor modifications. To simulate the various $\mathrm{pH}$ of GI tract in vivo, the continuous $\mathrm{pH}$ gradient solutions were used as dissolution medium. Firstly RTFA-OCDD-GN and RTFA extract, containing $6.0 \mathrm{mg}$ of RTFA, were placed in $900 \mathrm{~mL}$ of simulated gastric fluid ( $\mathrm{pH} 1.2$, containing $0.4 \% \mathrm{SDS}$ ) for $2 \mathrm{~h}$, and then placed in $900 \mathrm{~mL}$ of simulated small-intestinal fluid ( $\mathrm{pH} 6.8$, containing $0.4 \% \mathrm{SDS}$ ) for $4 \mathrm{~h}$, finally placed in $100 \mathrm{~mL}$ of simulated colonic fluid ( $\mathrm{pH} 7.4$, containing rat cecal contents and $0.4 \%$ SDS) for $4 \mathrm{~h}$ with continuous nitrogen gas supply. $2 \mathrm{~mL}$ samples were withdrawn at the given time intervals and immediately filtered through a $0.22-\mu \mathrm{m}$ membrane. The initial filtrate was discarded. Meanwhile an equal volume of prewamed medium was added. The RTFA concentration in each filtered sample was determined using HPLC. 
As demonstrated in the literature (Englyst, Hay, Macfarlane, 1987; Yang, Chu, Fix, 2002; Li et al., 2003), chitosan was degraded by the rat cecal contents because there is some microflora in the rat cedal contents generating $\beta$-glycosidase. Thus the $\mathrm{pH} 7.4$ phosphate buffer containing rat cecal contents was used to simulate the colonic fluid in this study. The rat cecal contents were obtained as following. In order to activate the $\beta$-glycosidase, five SD rats weighing 200-300 g were intragastrically administered with $2 \mathrm{~mL}$ chitosan solution $(2 \%, w / v)$ daily for 7 days. Rats were sacrificed by intraperitoneal injection with excessive ethyl carbamate at $30 \mathrm{~min}$ before the drug release study. The caecum was removed from the abdomen, and both ends were ligated, cut and weighed using the weight-bearing method. Then the cecal contents were suspended in 5\% $(w / w)$ bicarbonate buffer solution $\left(\mathrm{NaHCO}_{3} 9.240 \mathrm{~g}, \mathrm{Na}_{2} \mathrm{HPO}_{3} 7.125 \mathrm{~g}, \mathrm{NaCl}\right.$ $0.47 \mathrm{~g}, \mathrm{KCl} 0.45 \mathrm{~g}, \mathrm{CaCl}_{2} 0.073 \mathrm{~g}$ and $\mathrm{MgCl}_{2} 0.087 \mathrm{~g}$ were dissolved in $1,000 \mathrm{~mL}$ distilled water) previously bubbled with nitrogen gas. All these reactions were performed under nitrogen atmosphere. The activity of $\beta$-glycosidase contained in the rat cecal contents was verified by the dissolution test of core drug-load granules and granules sub-coated by chitosan in the $\mathrm{pH} 7.4$ phosphate buffer with the presence or absence of rat cecal contents (Figure 1).

\section{Pharmacodynamic study}

\section{RMM sample preparation}

RMM was powdered and then extracted with eight volumes of $40 \%$ ethanol for three times below $40^{\circ} \mathrm{C}$; the filtrates were pooled, concentrated and then freeze-dried and stored at $4^{\circ} \mathrm{C}$ until further analysis. This dried extract was used as the RMM samples. The contents of total AQs, free AQs and conjugated AQs determined by the method described in the Chinese

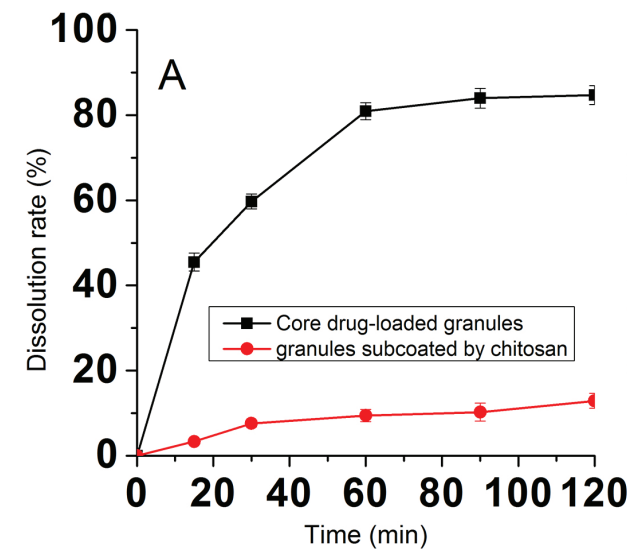

Pharmacopoeia (Pharmacopoeia, 2010) were 9.8\%, 4.8\% and $5.0 \%$ respectively. The ratio of conjugated AQs and free AQs in the extract was proximal 1:1 (w:w), which was consistent with that of RMM containing. The RMM samples were suspended with $0.5 \% \mathrm{CMC}-\mathrm{Na}$ before the oral administration.

\section{Purgative activity test}

A total of 70 healthy SD rats weighing 180-200 $\mathrm{g}$ were housed in a constant temperature environment $\left(21^{\circ} \mathrm{C}\right)$ with a 12 -h light/dark cycle. A standard diet with water was supplied during the period of acclimatization for 1 week. The rats were fasted for a night prior to the experiment. The rats were randomLy divided into seven groups with five males and five females in each group. The seven groups were administered orally RTFA-OCDD-GN (low-, medium- and high- dosage), RMM samples (low-, medium-, high- dosage) and normal saline, respectively. As demonstrated in the previous study, the RTFA contained in RMM samples barely produced purgative effect. Thus in the study the dosage of RTFA contained in RTFA-OCDDGN was equal to that of total conjugated AQs contained in RMM samples. The low-, medium- and high- dosage of orally administered RMM samples was $0.66,0.33,0.165$ $\mathrm{g} / \mathrm{kg}$ (equivalent to total conjugated AQs as 0.03056 , $0.01528,0.00764 \mathrm{~g} / \mathrm{kg}$ ) respectively, and those of RTFAOCDD-GN was $0.36,0.18,0.09 \mathrm{~g} / \mathrm{kg}$ (equivalent to RTFA as $0.03056,0.01528,0.00764 \mathrm{~g} / \mathrm{kg}$ ) respectively. The control group was orally administered with normal saline. The rats in seven groups were continuously monitored for $8 \mathrm{~h}$ immediately after the intragastric administration to obtain the defecation frequency and the conditions of stools. The conditions of stools were divided into five types as followings: normal, soft, loose, semi-liquid and watery. The diarrhea was evaluated with the defecation frequency of non-normal defecation.

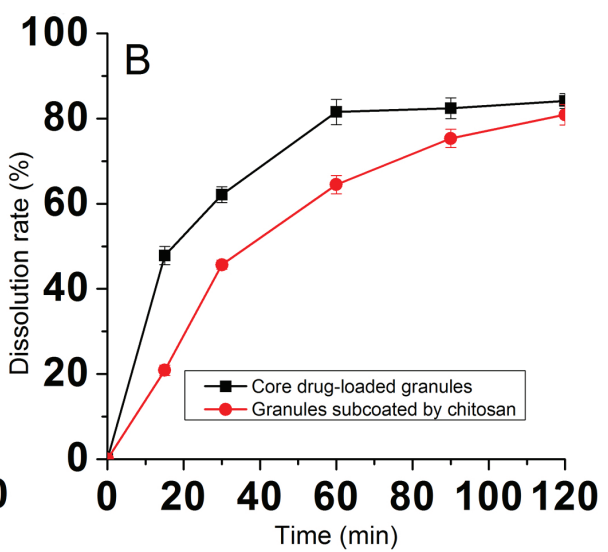

FIGURE 1 - The dissolution profiles of core drug-loaded granules and granules subcoated by chitosan in $\mathrm{pH} 7.4$ with the obsence of rat cecal contents (A) and presence of rat cecal contents (B). 


\section{Analytical method}

HPLC method was used to evaluate the dissolution rate of AQs from the different formulations. The HPLC analysis was conducted using Agilent 1200 (Agilent, USA). The separation was carried out on a reversed-phase Diamonsil $\mathrm{C}_{18}$ chromatographic column $(250 \mathrm{~mm} \times 4.6$ $\mathrm{mm}, 5 \mu \mathrm{m})$; the mobile phase was methanol and $1.1 \%$ perchloric acid aqueous solution $(80: 20, v: v)$; the flow rate was set at $1 \mathrm{~mL} / \mathrm{min}$; the wavelength was $254 \mathrm{~nm}$; and the column temperature was maintained at $30^{\circ} \mathrm{C}$. The standard curves of five free AQs were linear $(r>0.999)$ over the concentration range of 0.06624-6.624, 0.17933-17.933, $0.05088-5.088,0.04378-4.378$ and $0.02016-2.016 \mu \mathrm{g} /$ $\mathrm{mL}$, respectively. The inter-day and intra-day precisions were below $1.0 \%$. The average recovery was $99.5 \%$ with RSD of $1.4 \%$.

\section{Statistical analysis}

All values are expressed as the mean \pm standard deviation (SD). Significant differences between groups were analyzed by one-way ANOVA (SPSS 19.0 software). A $P$ value of less than 0.05 was considered as statistically significant.

\section{RESULTS AND DISCUSSION}

\section{Formulation Optimization}

\section{Core drug-loaded formulation}

The effect on the dissolution profile of different core drug-loaded formulations $(F 1, F 2$ and $F 3)$ was shown in Figure 2. In $F 1, F 2$ and $F$, the ratio of RTFA, MCC and PEG 6000 was $1: 2: 0.6,1: 4: 0.6$ and $1: 4: 1.2$ $(w: w: w)$, respectively. As shown in Figure 2A, in the $\mathrm{pH} 7.4$ phosphate buffer the accumulative dissolution rate of RTFA from the core drug-loaded granules was $80.40 \%, 82.94 \%$ and $81.40 \%$, respectively. This result showed that the ratio of RTFA, MCC and PEG screened in the formula had no significant effect on the dissolution rate of RTFA in the simulated colonic fluid. However, the core granules prepared by $F 1$ had a high viscosity, which was not advantageous for the following membrane coating process. In addition the drug loading in $F 2$ was superior to that of $F 3$. Thus the ratio of RTFA, MCC and PEG-6000 was selected as 1:4:0.6. The concentration of HPMC, as an adhesive, was optimized. As shown in Figure 2B, the dissolution rate of RTFA from the core drug-loaded granules prepared with 2\% HPMC was $82.4 \%$, which was superior to $1 \%$ and $3 \%$ HPMC.
Moreover, using 2\% HPMC containing 2\% PEG6000 as adhesive, the dissolution rate was high in comparison with $2 \%$ HPMC. Thus the 2\% HPMC containing 2\% PEG6000 was chosen as the adhesive. The Figure $2 \mathrm{C}$ showed that the dissolution profiles of three-batch core drug-loaded granules prepared with the optimized formulation (Table I) was fast and continuous with the accumulative release of $80 \%$ within $2 \mathrm{~h}$.

\section{Sub-coating formulation}

As shown in Figure 3, the dissolution profiles of sub-coating granules in simulated small intestinal fluid for $4 \mathrm{~h}$ were affected by the concentration of chitosan, the type and concentration of plasticizer, the amount of sub-coating formulation and the atomizing press. The dissolution profiles of the granules subcoated with chitosan at the concentration of $1.0 \%, 1.2 \%$ and $1.3 \%$ showed that the accumulative dissolution rate of RTFA were $28.49 \%, 19.50 \%$ and $19.84 \%$ in the simulated small intestinal fluid for $4 \mathrm{~h}$, respectively (Figure 3A). These results indicated that the dissolution rate of granules subcoated with chitosan of $1.0 \%$ was highest among three concentrations and the dissolution profiles of granules subcoated with chitosan of $1.2 \%$ and $1.3 \%$ showed no significant difference, demonstrating the release of RTFA was decreased significantly when the chitosan concentration reaching $1.2 \%$. However, the coating process of granules subcoated with chitosan at the concentration above $1.3 \%$ was very hard to achieve due to the high viscosity of chitosan. Thus, the chitosan concentration was optimized as $1.2 \%(w: v)$. In addition the accumulative dissolution rate of RTFA from granules subcoated with different plasticizers, such as glycerol, DEP and castor oil, was $19.35 \%, 28.82 \%$ and $29.86 \%$ respectively (Figure 3B). And the Figure 3C showed that the dissolution rate was reduced effectively with the glycerol concentration as $25 \%$ and $30 \%$. The glycerol, with the concentration of $25 \%$, was used as plasticizer in the optimized formulation due to the low viscosity. Moreover, when the amount of chitosan coating fluid was above $400 \mathrm{~mL}$, it was advantageous to obtain a satisfactory release profile (Figure 3D). And the Figure $3 \mathrm{E}$ showed that the atomizing press could affect the dissolution profile of RTFA-OCDD-GN and the accumulative dissolution rates of RTFA were $22.9 \%$ and $7.59 \%$ and $7.13 \%$ when the atomizing press was screened as $0.05,0.08$ and $1.0 \mathrm{Mpa}$, respectively. Thus, the parameter of atomizing press was chosen as $0.08 \mathrm{Mpa}$. According to the results above, the optimized chitosan sub-coating formulation was summarized as Table II. 

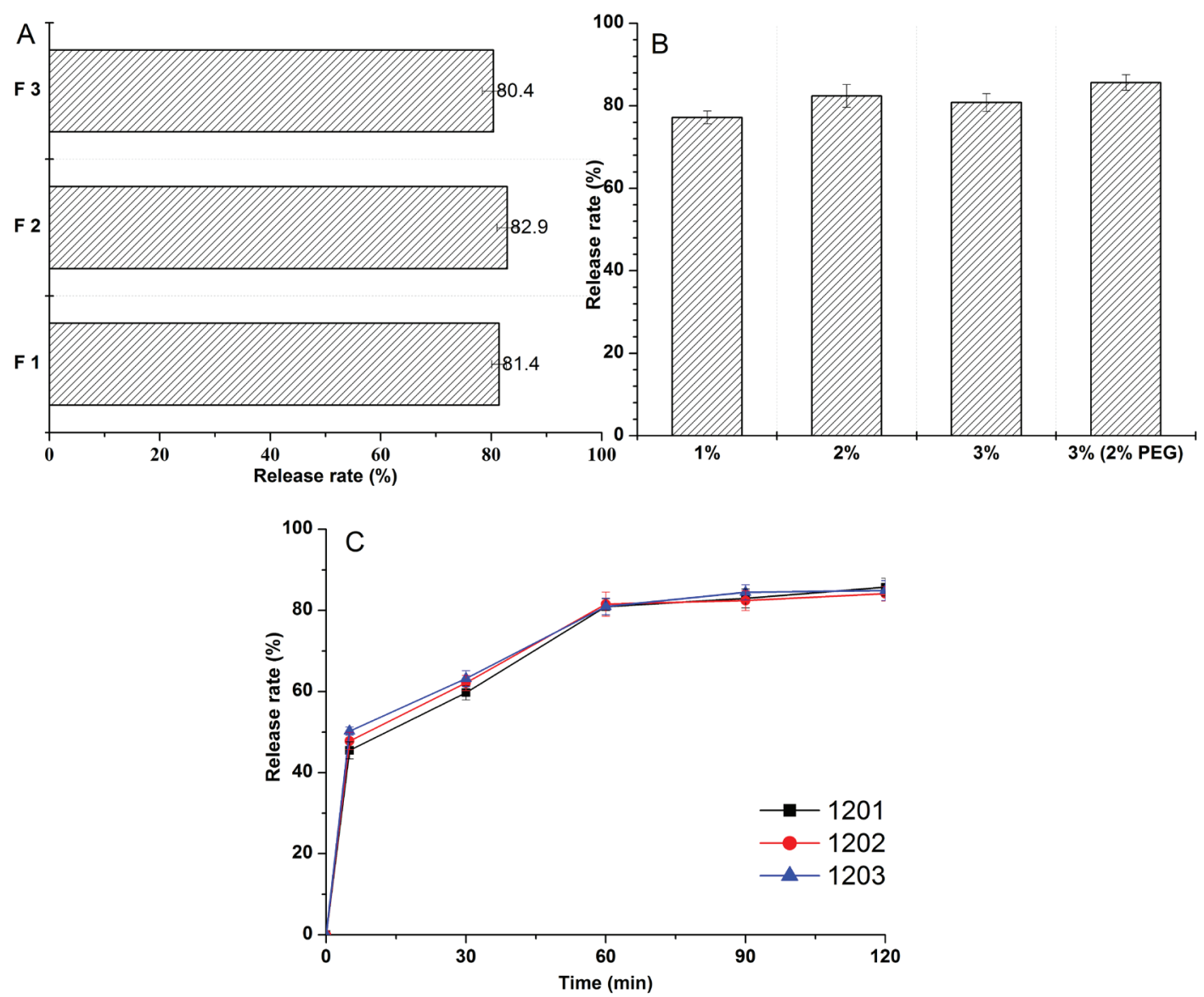

FIGURE 2 - The effect on dissolution rate of the ratio of RTFA, MCC and PEG (A) and the concentration of HPMC (B); and the release profile of RTFA from the core drug-loaded granules in a simulated colonic fluid (pH 7.4) containing rat caecal contents (C).

\section{Enteric coating formulation}

The ratio and concentration of Eudragit S100 and L100, the type and concentration of plasticizer, the amount of enteric coating fluid and the atomizing press were screened to optimize the enteric coating formulation. The dissolution profiles of different formulations in simulated gastric fluid ( $\mathrm{pH} 1.2)$ for $2 \mathrm{~h}$ and simulated small intestinal fluid $(\mathrm{pH}$ 6.8) for $4 \mathrm{~h}$ were shown in Figure 4. The Figure 4A showed that the accumulative dissolution rate of RTFA was significantly affected by the ratio of Eudragit S100 and L100. The dissolution rate of RTFA was increased with the increasing Eudragit L100 in enteric coating formulation. The concentration of Eudragit S100 in the enteric coating formulation could impact the dissolution profile (Figure 4B). When it was 3\%, the dissolution profile in the simulated gastric fluid was not accepted by the criteria documented in Chinese Pharmacopoeia for the enteric performance test. However, the release profiles showed that there was no drug released in the first $2 \mathrm{~h}$ with the concentration of Eudragit S100 between 5\% and $6 \%$. These results indicated that Eudragit S100 with the concentration above $5 \%$ possessed satisfactory film forming property and the ability to prevent the release of RTFA from the coating granules in the simulated gastric fluid. However, the Eudragit S100 concentration in the enteric coating formulation was optimized as $5 \%$ due to the advantage for the coating process by fluid bed. Different types of plasticizers have different effects on the dissolution profile (Figure 4C). The DEP could reduce significantly the dissolution rate of RTFA in comparison with PEG6000 and castor oil. Moreover, the dissolution profiles of granules coated by enteric coating formulation showed no significant difference with the DEP used as plasticizer at the concentration of $20 \%$ and $25 \%(w / w)$ (Figure 4D). However, it was found that the granules adhered together easily in the coating process when the amount of plasticizer in the enteric coating fluid was increased up to $25 \%$. Hence the concentration of DEP was optimized as $20 \%$. Furthermore, more than $300 \mathrm{~mL}$ of Eudragit S100 enteric coating formulation and above $0.05 \mathrm{Mpa}$ atomizing press were optimized to obtain the satisfactory dissolution profile (Figure 4E-F). According to the results above, the optimized enteric coating formulation was summarized as Table III. 

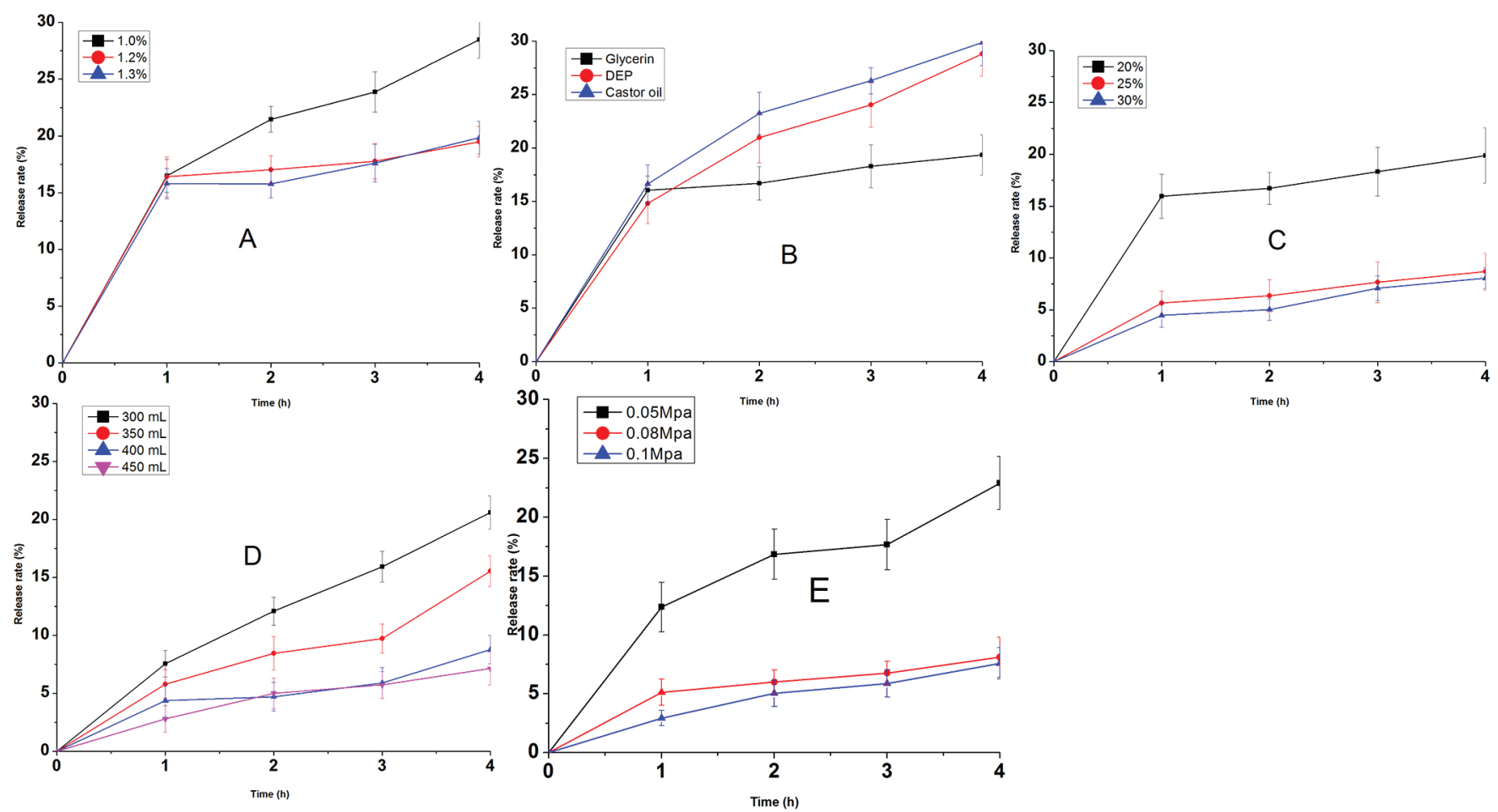

FIGURE 3 - The effect on dissolution rate in a simulated small-intestinal fluid (pH 6.8) of the chitosan concentration (A), the type of plasticizers (B), the concentration of plasticizers (C), the amount of coating fluid (D) atomizing press (E).
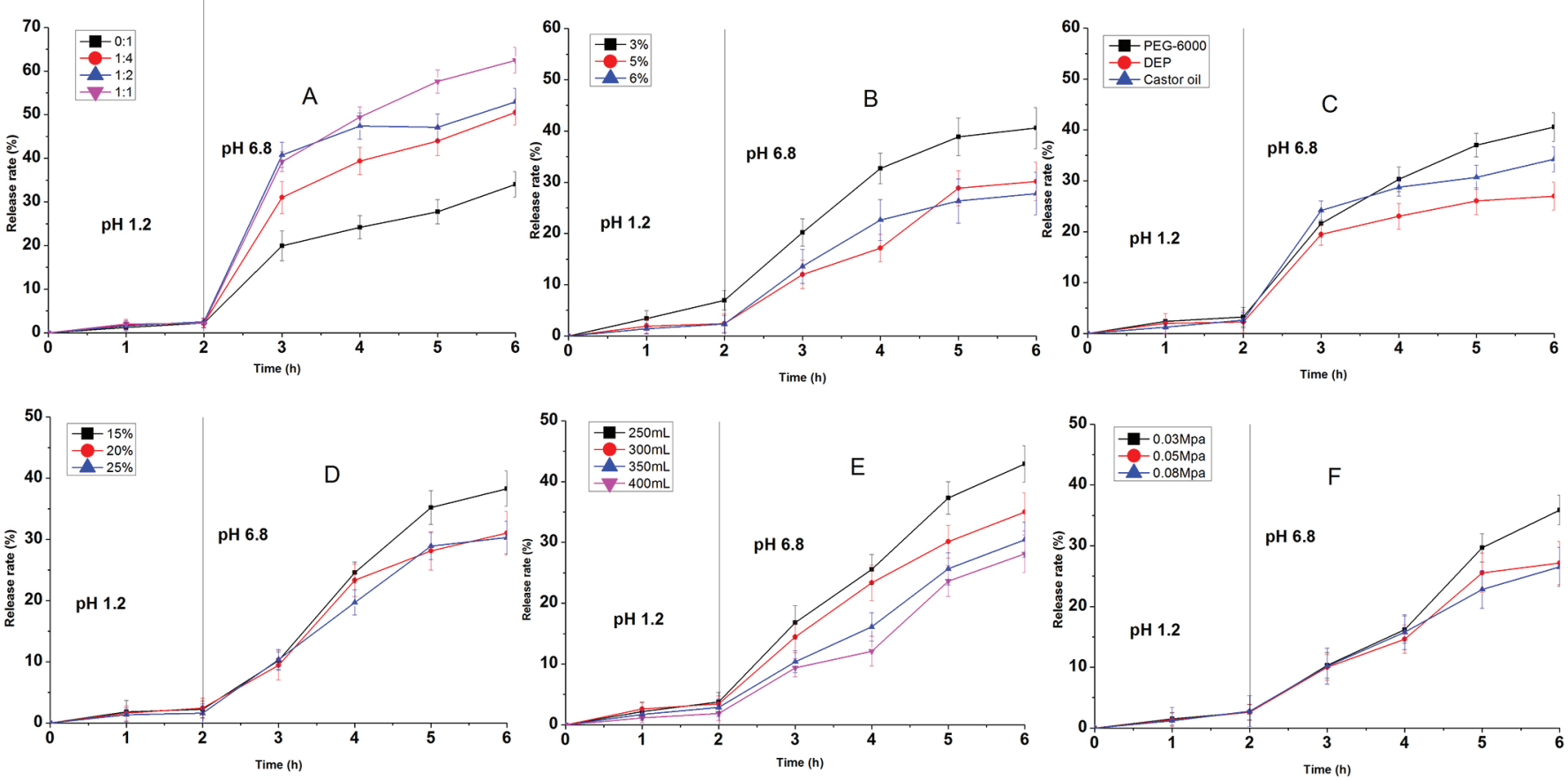

FIGURE 4 - The effect on dissolution rate in a simulated gastric fluid (pH 1.2) and a simulated small-intestinal fluid (pH 6.8) of the ratio of Eudragit S100 and L100 (A), the Eudragit S100 concentration (B), the type of plasticizers (C), the concentration of plasticizers (D), the amount of coating fluid (E) atomizing press (F).

The interaction of chitosan formulation and Eudragit S100 formulation

According to factorial design of RTFA-OCDD-GN, the factor combinations yielded a variety of calculated mean $L$ values. The values of $L$ are shown in Table IV. The values of $L$ were in the range of 19.42-12.27 for runs 
TABLE IV - Experimental runs and the determined values of $L$

\begin{tabular}{cccccc}
\hline Formulation NO. & $\begin{array}{c}\text { The amount of } \\
\text { chitosan }(\mathbf{m L})\end{array}$ & $\begin{array}{c}\text { The amount of } \\
\text { resin }(\mathbf{m L})\end{array}$ & $\boldsymbol{L}_{\boldsymbol{1}}$ & $\boldsymbol{L}_{\mathbf{2}}$ & $\boldsymbol{L}$ \\
\hline 1 & 400 & 300 & 13.54 & 2.94 & 19.42 \\
2 & 400 & 340 & 11.35 & 2.96 & 17.31 \\
3 & 400 & 380 & 6.57 & 4.31 & 15.19 \\
4 & 420 & 300 & 7.35 & 4.00 & 18.70 \\
5 & 420 & 340 & 6.81 & 3.00 & 12.81 \\
6 & 420 & 380 & 6.61 & 3.00 & 12.61 \\
7 & 450 & 300 & 8.92 & 2.99 & 14.90 \\
8 & 450 & 340 & 6.45 & 2.97 & 12.38 \\
9 & 450 & 380 & 6.32 & 2.97 & 12.27 \\
\hline
\end{tabular}

The values of $\mathrm{L}_{1}, \mathrm{~L}_{2}$ and $\mathrm{L}$ are expressed as mean.

1 to 9 . The lowest value for $L$ was achieved by run 9 . However, the variance of $L$ was not significant for runs 5 to 9 . Thus $420 \mathrm{~mL}$ of chitosan formulation and $340 \mathrm{~mL}$ of Eudragit S100 formulation were chosen in the study due to the availability for the coating process. Analysis of variance (ANOVA) according to the data of $L$ value is shown in Table V. According to ANOVA, all $P$-values listed presented a significant effect of the independent factors on $L$ values and the interaction of $A$ and $B$ was presented $(P<0.05)$. These results indicated that the chitosan formulation and Eudragit S100 formulation produced the synergetic effect on the dissolution profile of RTFA-OCDD-GN, contributing to improve the stability and reproducibility of RTFA-OCDD-GN.

TABLE V - Variance analysis

\begin{tabular}{lccccc}
\hline Factors & SS & DF & MS & F & P \\
\hline Total & 190.399 & 26 & & & \\
A & 78.208 & 2 & 39.104 & 6269.656 & $<0.01$ \\
B & 94.829 & 2 & 47.415 & 7602.121 & $<0.01$ \\
AB & 17.249 & 4 & 4.312 & 691.384 & $<0.01$ \\
Error & 0.112 & 18 & 0.006 & & \\
\hline
\end{tabular}

\section{In vitro dissolution}

In the present study, the dissolution test of RTFAOCDD-GN were performed successively in the simulated gastric fluid ( $\mathrm{pH} 1.2)$ for $2 \mathrm{~h}$, and then in the simulated small-intestinal fluid ( $\mathrm{pH} \mathrm{6.8)} \mathrm{for} 4 \mathrm{~h}$, and finally in the simulated colonic fluid ( $\mathrm{pH} 7.4$ ) with or without the presence of rat cecal contents for $4 \mathrm{~h}$. The dissolution profiles of RTFA-OCDD-GN and RTFA extract was shown in Figure 5. As shown in Figure 5A, the accumulative dissolution rate of RTFA from RTFA-OCDD-GN was $9.0 \%$ in the simulated gastric and small-intestinal fluid for $6 \mathrm{~h}$, and it was $82.6 \%$ in the simulated colonic fluid containing rat cecal contents for $4 \mathrm{~h}$; however it was only about $10 \%$ in the simulated colonic fluid without the presence of rat cecal contents. In addition, the dissolution profile of RTFA extract showed that the accumulative dissolution rate of RTFA was about $80 \%$ in the simulated gastric fluid for $2 \mathrm{~h}$. Although there were slight RTFA released from RTFA-OCDD-GN in the simulated gastric and small intestinal fluid, the risk of without releasing any drug in the GI tract was attenuated, as found in the study of Ashford et al. (1993). Thus, these results indicated that the RTFA-OCDD-GN was prepared successfully with chitosan and Eudragit S100 by the double-layer coating process. Moreover, the RTFA-OCDD-GN achieved the objectives of colon-specific delivery system: (i) prevention of premature drug release in the GI tract; and (ii) rapid drug release in the colonic fluid. The colon-specific release characterization of RTFA-OCDD-GN was attributed to the release mechanism of Eudragit S100 and chitosan. Eudragit S100, a pH-dependent polymer, can dissolve at and above $\mathrm{pH}$ 7.0. Chitosan, a weak basic copolymer of $\beta$ - $(1,4)-2$-acetamido-2-deoxy-D-glucopyranose and 2-amino-2-deoxy-D-glucopyranose, undergo the hydrolysis of their glycoside bonds in the colon. Hence the mechanism that the RTFA released from the RTFAOCDD-GN was described in detail as followings: during the RTFA transporting in the whole gastrointestinal tract the RTFA is scarcely released in the stomach, and slightly released in the small intestinal due to the dissolution of outer Eudragit S100 coating layer, and finally in the colon quickly released due to the degradation of chitosan. 
In addition, the Figure 5B showed the reproducibility of dissolution test of three batches RTFA-OCDD-GN. As shown in Figure 5B, the dissolution profiles of three batches RTFA-OCDD-GN were basically similar with the value of $f_{2}$ as between 50 and 100 , indicating the preparation process of RTFA-OCDD-GN reproduced well.

\section{Pharmacodynamic study}

In the previous study, it was demonstrated that the RTFA in a common dosage scarcely produced purgative effect (the data not shown). Moreover the rhubarb used in the TCM is in the form of either all or partial crude powder. Thus the RTFA-OCDD-GN ability to improve the purgative activity was compared with RMM samples. The comparison of RTFA-OCDD-GN and RMM samples on the defecation frequency and consistence of stool was shown in Figure 6. Compared with the control group, the defecation frequency was increased significantly in the RMM samples group (containing low-, middle- and high-dosage subgroups) and RTFA-OCDD-GN group (containing low-, middle- and high-dosage subgroups) $(P<0.05)$. In addition, there was no significant difference observed between the RMM samples group and RTFAOCDD-GN group (in all dosages, $P>0.05$ ). However, the consistence of faeces in RTFA-OCDD-GN groups was different from that of RMM samples groups. As shown in Figure 6B, the frequency of semi-liquid and watery defecation in the RTFA-OCDD-GN groups (medium- and high- dosage) was decreased significantly in comparison with RMM samples groups (medium- and high- dosage) $(p<0.05)$. The reason speculated was that RTFA-OCDDGN was prepared with RTFA extract, in which AQs existed in free form and thereby the free AQs only produced mild purgation. Thus the purgative study results indicated that RTFA-OCDD-GN could produce corresponding purgative effect in low oral dosage compared with RMM samples, contributing to reduce the risk of accumulation for AQs in vivo; in addition RTFA-OCDD-GN reduced the incidence frequency of diarrhea. Moreover, as demonstrated in our previous study the purgative effect caused by RTFA extract with the dosage used in this study was significantly lower than that of RMM (the data not shown in the paper). Therefore, these results also suggested that the RTFAOCDD-GN could basically delivery all RTFA to the colon to release after directly oral administration. Indeed, the pharmacokinetic study of RTFA-OCDD-GN confirmed that RTFA-OCDD-GN could prevent RTFA from releasing and being adsorbed in the stomach and small intestine and delivery RTFA to colon, which has been published in another paper (Zhang et al., 2015).

\section{CONCLUSION}

In the present study, we successfully prepared the RTFA-OCDD-GN through a double-layer coating process using chitosan as an inner layer and Eudragit S100 as an outer layer. The accumulative dissolution rate of RTFA-OCDD-GN was about $83 \%$ in the simulated colonic fluid with the presence of rat cecal contents, whereas it was about only $9.0 \%$ in the simulated gastric and small-intestinal fluids. These results indicated that
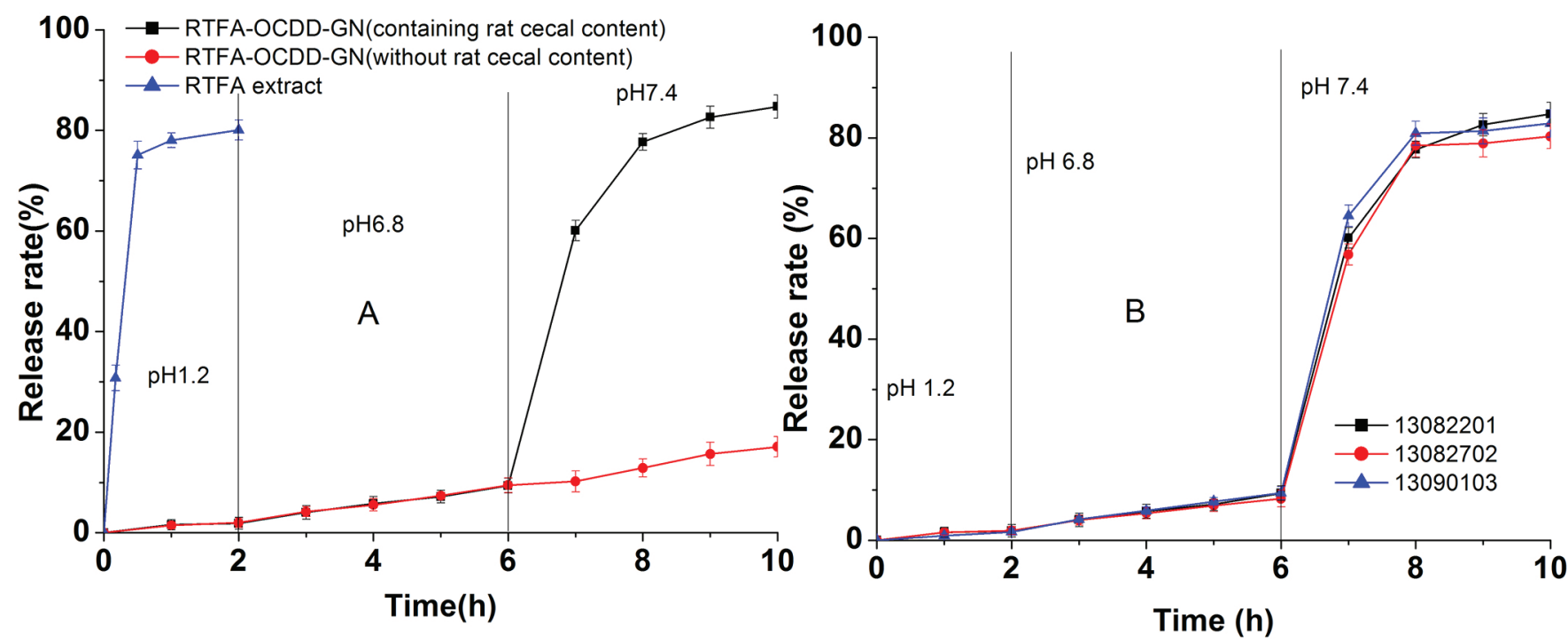

FIGURE 5 - The dissolution profiles of the core drug-load granules in pH 1.2 and RTFA-OCDD-GN in a simulated gastric fluid $(\mathrm{pH}$ 1.2) and a simulated small-intestinal fluid ( $\mathrm{pH}$ 6.8) and a simulated colonic fluid (pH 7.4) in the presence or absence of rat cecal contents (A), and the dissolution test reproducibility of three batches RTFA-OCDD-GN (B). 

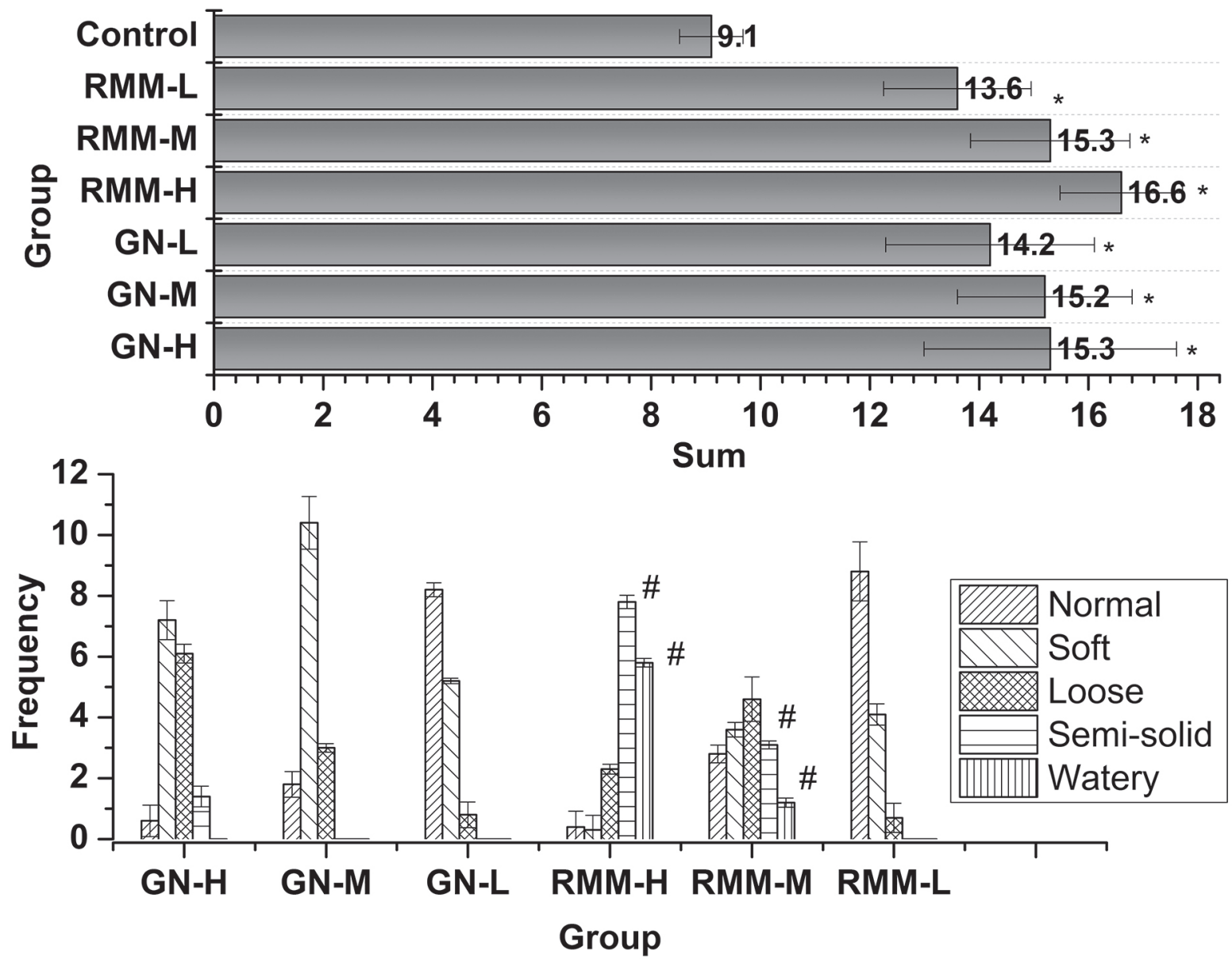

FIGURE 6 - The comparison on defecation frequency and consistance of faeces of RMM and RTA-OCDD-GN groups after oral administration to rats. The data were expressed as mean \pm S.D. $(\mathrm{n}=10) .{ }^{*} P<0.05$, compared with the normal group; ${ }^{\sharp} P<0.05$, compared with the RTFA-OCDD-GN group.

RTFA-OCDD-GN could release specifically in the colon. Moreover the RTFA-OCDD-GN and RMM samples could produce corresponding purgative activity, but the dosage of RTFA-OCDD-GN was only about half of that of RMM samples. Therefore, the RTFA-OCDD-GN could be a useful drug delivery system to improve the purgative effect and reduce the oral dosage for AQs.

\section{CONFLICT OF INTEREST}

The authors declare that there is no conflict of interests regarding the publication of this paper.

\section{ACKNOWLEDGEMENTS}

This work was financially supported by the Key Discipline Construction Projects of Higher School; the National Natural Science Foundation of China (No. 81073146); Science and Technology Research Project of Administration of Traditional Chinese Medicine of Hebei
Province (No. 2016188); Medical Science Research Key Project of Health and Family Planning Commission of Hebei Province (No. 2060311).

\section{REFERENCE}

Ashford M, Fell JT, Attwood D, Sharma H, Woodhead PJ. An in vivo investigation into the suitability of $\mathrm{pH}$ dependent polymers for colonic targeting. Int J Pharm. 1993;95(1-3):193-199.

Englyst HN, Hay S, Macfarlane GT. Polysaccharide breakdown by mixed populations of human faecal bacteria. FEMS Microbiol Ecol. 1987;45(3):163-171.

Fu XS, Cheng F, Liu XH, Xu H, Zhou YZ. Progress in research of chemical constituents and pharmacological actions of Rhubarb. Chinese J New Drugs. 2011;20(16):1534-1539. 
Italia JL, Bhatt DK, Bhardwaj V, Tikoo K, Ravi Kumar MNV. PLGA nanoparticles for oral delivery of cyclosporine: Nephrotoxicity and pharmacokinetic studies in comparison to Sandimmune Neoral ${ }^{\circledR}$. J Control Release. 2007;119(2):197-206.

Kaur K, Kim K. Studies of chitosan/organic acid/Eudragit $($ RS/ RL-coated system for colonic delivery. Int J Pharm. 2009;366(12):140-148.

Keiyu O, Mikito H, Akihisa M, Tomoya M, Yohei M. Toxicological effect of emodin in mouse testicular gene expression profile. J Appl Toxicol. 2011;31(8):790-800.

Khan MZI, Prebeg Z, Kurjakovic N. A pH-dependent colon targeted oral drug delivery system using methacrylic acid copolymers I. Manipulation of drug release using Eudragit ${ }^{\circledR}$ L100-55 and Eudragit ${ }^{\circledR}$ S100 combinations. J Control Release. 1999;58(2):215-222.

Li GF, Chen JH, Jiang LX, Chen ZL, Yan Y, Lai ZS, Zhou ZT. Study on colon-specific delivery of HPMCP-coated mesalazine chitosan capsules in vitro. Chin Pharm J. 2003;38:601-604.

Liu CZ, Liu XG, Chen DW, Bi KS. Studies on detecting method of San-huang rapid-release tablets. Chin J Pharm Anal. 2005;25:929-931.

Liu CZ, Liu XG, Tong JM, Chen DW, Bi KS. Design and evaluation of San-huang dispersible tablet--an efficient delivery system for Traditional Chinese Medicine. Pharm Dev Technol. 2009;14(5):506-515.

Liu XG, Cui YH, Chen DW, Li ZS, Chang JH, Liu CZ. Study on in situ rats stomach and intestinal absorption of the total anthraquinones of rhubarb. Zhongguo Yi Yuan Yao Xue Za Zhi. 2011;31(3):188-191.

Min GN, Wu XA, Qi YL, Min GT, Sun XD, Wang XH. Preliminary study on cathartic effects of free anthraquinone in rhubarb. Lanzhou Da Xue Xue Bao (Med Sci). 2008;34:41-43.

Moore JW, Flanner HH. Mathematical comparison of dissolution profiles. Pharm Tech. 1996;20:64-74.

National Toxicology Program (NTP). Toxicology and carcinogenesis studies of emodin (CAS NO: 1518-82-1) feed studies in F344/N rats and B6C3F1 mice. Natl Toxical Program Tech Rep Ser. 2001;493:1-2781.
Orienti I, Cerchiara T, Luppi B, Bigucci F, Zuccari G, Zecchi $\mathrm{V}$. Influence of different chitosan salts on the release of sodium diclofenac in colon-specific delivery. Int J Pharm. 2002;238(12):51-59.

Pharmacopoeia of People's Republic of China. Beijing: Chemical Industry Press; 2010. v.1, 22 p.

Piao ZZ, Lee MK, Lee BJ. Colonic release and reduced intestinal tissue damage of coated tablets containing naproxen inclusion complex. Int J Pharm. 2008;350(1-2):205-211.

Rauwald HW. Herbal laxatives: Influence of anthronesanthraquinones on energy metabolism and ion transport in a model system. Acs Symposium. 1998;691(2):97-116.

Sun YQ, Xiao XH, Ma YG, Xing XY, Yan D. Study on dynamic variation patterns of anthraquinones of radix et rhiroma rhei at different decocting time. Pharm J Chin PLA. 2006;22:281-283.

Tozaki H, Komoike J, Tada C, Maruyama T, Terabe A, Suzuki $\mathrm{T}$, et al. Chitosan capsules for colon-specific drug delivery: improvement of insulin absorption from the rat colon. J Pharm Sci. 1997;86(9):1016-1021.

Tozaki H, Odoriba T, Okada N, Fujita T, Terabe A, Suzuki T, et al. Chitosan capsules for colon-specific drug delivery: enhanced localization of 5-aminosalicylic acid in the large intestine accelerates healing of TNBS-induced colitis in rats. J Control Release. 2002;82(1):51-61.

Wang YQ. Ingredients analysis of traditional Chinese medicine. Gui Yang: Guizhou Sscience and Technology Press; 1991. p. 505-507.

Wang JB, Ma YG, Zhang P, Jin CH, Sun YQ, Xiao XH, et al. Effect of processing on the chemical contents and hepatic and renal toxicity of rhubarb studied by canonical correlation analysis. Acta Pharmacol Sin. 2009;44(8):885-890.

Wu XA. Opinion of colon-targeting delivery about rhubarb extract as a purgative. Zhongguo Zhong Yao Za Zhi. 2002;27(1):75-77.

Yamauchi K, Shinano K, Nakajima K, Yaqi T, Kuwano S. Metabolic activation of sennosid $\mathrm{C}$ in mice; synergistic action of anthrones. J Pharm Pharmacol. 1992;44(12):973-976. 
Yang LB, Chu JS, Fix JA. Colon-specific drug delivery: new approaches and in vitro/in vivo evaluation. Int J Pharm. 2002;235(1-2):1-15.

Yang H, Xu LN, He CY, Liu X, Fang RY, Ma TH. CFTR chloride channel as a molecular target of anthraquinone compounds in herbal laxatives. Acta Pharmacol Sin. 2011;32(6):834-839.

Yang YM, Wang P, Zhang Y. A comparative study on the absorption kinetics parameters of rhubarb free anthraquinones between normal dogs and dogs with severe acute pancreatitis. Zhongguo Zhong Xi Yi Jie He Za Zhi. 2012;32(4):494-498.
Yi QN, Wang JB, Kong WJ, Zhao YL, Yang HY, Dai CM, Fang, F, et al. The diarrhoeogenic and antidiarrhoeal bidirectional effects of rhubarb and its potential mechanism. J Ethnopharmacol. 2011;133(3):1096-1102.

Zhang L, Chang JH, Zhang BQ, Liu XG, Liu P, Xue HF, et al. The pharmacokinetic study on the mechanism of toxicity attenuation of rhubarb total free anthraquinone oral colonspecific drug delivery system. Fitoterapia. 2015;104;86-96.

Received for publication on $05^{\text {th }}$ March 2017 Accepted for publication on $25^{\text {th }}$ February 2018 